\title{
A Competitive Two-Agent Scheduling Problem on Parallel Machines with Release Dates and Preemption
}

\author{
Yawei Qi ${ }^{1}$ and Long Wan ${ }^{2}$ \\ ${ }^{1}$ School of Information Technology, Jiangxi Key Laboratory of Data and Knowledge Engineering, \\ Jiangxi University of Finance and Economics, Nanchang, Jiangxi 330013, China \\ ${ }^{2}$ School of Information Technology, Jiangxi University of Finance and Economics, Nanchang, Jiangxi 330013, China
}

Correspondence should be addressed to Yawei Qi; ivey09@163.com

Received 12 August 2013; Accepted 6 October 2013

Academic Editor: Yunqiang Yin

Copyright (C) 2013 Y. Qi and L. Wan. This is an open access article distributed under the Creative Commons Attribution License, which permits unrestricted use, distribution, and reproduction in any medium, provided the original work is properly cited.

\begin{abstract}
We consider a competitive two-agent scheduling problem on multiple identical machines with release dates and preemption. In the scheduling model, there are two agents $a$ and $b$ each having their own job sets $\mathscr{F}_{a}=\left\{J_{1}^{a}, \ldots, J_{n_{a}}^{a}\right\}$ and $\mathscr{J}_{b}=\left\{J_{1}^{b}, \ldots, J_{n_{b}}^{b}\right\}$, respectively. Each job $J_{j} \in \mathscr{J}_{a} \cup \mathscr{J}_{b}$ has a release date $r_{i}$ and the $n=n_{a}+n_{b}$ jobs need to be preemptively scheduled on $m$ identical machines. For $m=2$, we show that the trade-off curve of all the Pareto optimal points can be characterized in polynomial time. When $m$ is input, we show that $P\left|r_{j}, \operatorname{pmtn}\right| L_{\max }^{a}: L_{\max }^{b} \leq Q$ can be solved in strongly polynomial time.
\end{abstract}

\section{Introduction and Problem Formulation}

In recent years, multiagent scheduling problems are under extensive research. A multiagent scheduling problem means that there are multiple agents which must compete to perform their own tasks on the common processing resource. Each agent wants to optimize his/her own objective function. The objective function considered in this paper is to minimize the maximum lateness of the jobs. First, let us briefly recall the history of the classic scheduling problems of minimizing the maximum lateness of the jobs that is, there is only one agent in such a problem. Horn [1] considered the problem of scheduling the jobs preemptively on identical machines with release dates and deadlines and showed that it can be determined in strongly polynomial time if the problem has a feasible schedule by reducing it to a network flow problem which is well known to be solved in strongly polynomial time. Sahni [2] presented a faster algorithm to determine if the problem with identical release dates has a feasible schedule. Furthermore, Sahni and Cho [3] showed that it also can be determined in strongly polynomial time if the problem on related machines has a feasible schedule. Lawler and Labetoulle [4] proved that the feasibility problem on unrelated machines can be settled in weakly polynomial time by means of linear programming. Labetoulle et al. [5] studied the problems of scheduling the jobs on the parallel machines preemptively to minimize the maximum lateness of the jobs with release dates. They gave a strongly polynomial-time algorithm to solve the problem on identical machines which is based on the same network flow structure introduced by Horn [1] and a weakly polynomial-time algorithm to solve the problem on uniform machines in terms of the polymatroidal network flow model introduced by Lawler and Martel [6]. For the latter, a similar result can be found in Martel [7]. The multiagent scheduling models were initially introduced by Baker and Smith [8] and Agnetis et al. [9]. Their research focused on the problems of nonpreemptively scheduling the jobs which belong to two agents on a single machine. Agnetis et al. [10] investigated the multiagent single machine problem of finding a nonpreemptive schedule in which the cost of each agent does not exceed a given threshold value which is also studied in Cheng et al. [11]. Cheng et al. [12] considered the feasibility model of multiagent scheduling on a single machine for which each agent competes to minimize the total weighted number of his/her own tardy jobs and showed that the problem is strongly NP-hard. For more papers about the multiagent problems of scheduling the jobs nonpreemptively on a single machine, the readers are referred to Yuan et al. 
[13], Ng et al. [14], and Mor and Mosheiov [15]. Leung et al. [16] which initiated the preemptively multiagent scheduling problems investigated the two-agent scheduling problems of scheduling the jobs preemptively on a single machine or identical machines. Yuan et al. [17] studied a competitive two-agent scheduling problem on a single machine with release dates and preemption for which the objective of each agent is to minimize the maximum lateness and showed that all Pareto optimal points can be found in strongly polynomial time. Wan et al. [18] investigated the same twoagent scheduling model for which one agent's objective is to minimize the maximum lateness and the other agent's objective is to minimize the total completion time of his/her jobs. They proved that the problem is NP-hard in the ordinary sense by means of reduction from even-odd partition which is well known to be ordinarily NP-hard [19].

The problems in the paper are stated as follows. There are two agents $a$ and $b$ each having their own job sets $\mathscr{J}_{a}=$ $\left\{J_{1}^{a}, \ldots, J_{n_{a}}^{a}\right\}$ and $\mathscr{J}_{b}=\left\{J_{1}^{b}, \ldots, J_{n_{b}}^{b}\right\}$, respectively. We make the assumption that $\mathscr{J}_{a} \cap \mathscr{J}_{b}=\emptyset$. The jobs in $\mathscr{J}_{a}$ are called $a$-jobs and the jobs in $\mathscr{F}_{b}$ are called $b$-jobs. Each job $J_{j} \in \mathscr{J}_{a} \cup \mathscr{J}_{b}$ has a release date $r_{j}$, a due date $d_{j}$, and a processing time $p_{j}$. And the $n=n_{a}+n_{b}$ jobs need to be preemptively scheduled on $m$ identical machines. Two agents have the same objective of minimizing the maximum lateness. We use the following notation throughout this paper.

(i) $p_{j}^{x}$ is the processing time of job $J_{j}^{x}, x \in\{a, b\}$.

(ii) $r_{j}^{x}$ is the release date of job $J_{j}^{x}, x \in\{a, b\}$.

(iii) $d_{j}^{x}$ is the due date of job $J_{j}^{x}, x \in\{a, b\}$.

(iv) $C_{j}^{x}$ is the completion time of job $J_{j}^{x}, x \in\{a, b\}$.

(v) $L_{j}^{x}=C_{j}^{x}-d_{j}^{x}$ is the lateness of job $J_{j}^{x}, x \in\{a, b\}$.

(vi) $L_{\max }^{x}=\max \left\{L_{j}^{x}: 1 \leq j \leq n_{x}\right\}$ is the maximum lateness of (the jobs of) agent $x, x \in\{a, b\}$.

A schedule $\sigma$ is called Pareto optimal if there is no schedule $\rho$ such that $L_{\max }^{a}(\rho) \leq L_{\max }^{a}(\sigma), L_{\max }^{b}(\rho) \leq L_{\max }^{b}(\sigma)$, and at least one inequality strictly holds, that is, a schedule is Pareto optimal for any schedule; if it is better for one agent, then it must be worse for the other agent. We say $\left(L_{\max }^{a}(\sigma), L_{\max }^{b}(\sigma)\right)$ is a Pareto optimal point if schedule $\sigma$ is a Pareto optimal schedule. The first problem we consider is to find all the Pareto optimal points and a corresponding schedule for each Pareto optimal point when $m=2$ and all the jobs are released at 0 . The second problem we consider is to minimize the maximum lateness of agent $A$ with the maximum lateness of agent $B$ bounded by a given threshold $Q$ when $m$ is input.

By use of the well-known three-field notation [20], the first and second problems can be formulated as follows.

(i) The first problem: $P 2|\operatorname{pmtn}|\left(L_{\max }^{a}: L_{\max }^{b}\right)$, which is a Pareto optimization problem seeking to minimize $L_{\max }^{a}$ and $L_{\max }^{b}$ simultaneously.

(ii) The second problem: $P\left|r_{j}, \operatorname{pmtn}\right| L_{\text {max }}^{a}: L_{\max }^{b} \leq Q$.
The rest of the paper is organized as follows. In Section 2, we show that the tradeoff curve of the Pareto optimal points can be characterized in strongly polynomial time for $P 2|\operatorname{pmtn}|\left(L_{\max }^{a}: L_{\max }^{b}\right)$. Section 3 gives a polynomial time algorithm to solve Problem $P\left|r_{j}, \operatorname{pmtn}\right| L_{\text {max }}^{a}: L_{\max }^{b} \leq \mathrm{Q}$. We draw some conclusions and present some further research.

\section{2. $P 2|\operatorname{pmtn}|\left(L_{\max }^{a}: L_{\max }^{b}\right)$}

First, let us state a feasibility problem of scheduling $n$ jobs preemptively on two identical machines with the deadlines and give a characterization of feasibility. Let $\mathscr{J}=\left\{J_{1}, J_{2}, \ldots, J_{n}\right\}$ be the set of the jobs and $d_{j}$ and $p_{j}$ are the deadline and processing time of job $J_{j}$, respectively; $j=1,2, \ldots, n$. The problem is denoted by $P 2 \mid$ pmtn; $d_{j} \mid$ - in terms of the threefield notation. Without loss of generality, we assume that the jobs are ordered by $d_{1} \leq d_{2} \leq \cdots \leq d_{n}$. Assume that there are total $k$ different deadlines and $d_{(1)}, d_{(2)}, \ldots, d_{(k)}$ with $d_{(1)}<d_{(2)}<\cdots<d_{(k)}$ are the $k$ different deadlines. Denote by $g^{(i)}$ the set of the jobs of deadline $d_{(i)}, i=1,2, \ldots, k$. Let $r_{0}=0, r_{1}, \ldots, r_{k}=n$ be the $k+1$ numbers such that $\mathscr{J}^{(i)}=\left\{J_{r_{i-1}+1}, J_{r_{i-1}+2}, \ldots, J_{r_{i}}\right\}, i=1,2, \ldots, k$. We assume that $d_{0}=0$ and $J_{r_{i-1}+1}$ is the largest job of $\mathcal{J}^{(i)}$ for convenience, $i=1,2, \ldots, k$.

Before we present the characterization of feasibility of $P 2|\operatorname{pmtn}|\left(L_{\max }^{a}: L_{\max }^{b}\right)$, we first state a well-known result from [2].

Algorithm Sahni. Consider the following steps.

Step 1. $i=1, L_{1}=L_{2}=0$.

Step 2. Find a unscheduled job $J_{j}$ of $\mathscr{J}^{(i)}$. If $p_{j} \geq d_{(i)}-L_{1}$, then return infeasibility. Otherwise, we schedule job $J_{j}$. If $p_{j} \leq$ $d_{(i)}-L_{2}$, then we assign time interval $\left[L_{2}, L_{2}+p_{j}\right]$ to $J_{j}$ on machine 2 and reset $L_{2}:=L_{2}+p_{j}$; else, we assign time interval $\left[L_{2}, d_{(i)}\right]$ on machine 2 and time interval $\left[L_{1}, L_{1}+L_{2}+p_{j}-d_{(i)}\right]$ on machine 1 to $J_{j}$ and reset $L_{2}:=d_{(i)}, L_{1}:=L_{1}+L_{2}+p_{j}-d_{(i)}$.

Step 3. If all the jobs of $\mathscr{J}^{(i)}$ are scheduled, then reset $i:=i+1$; else, go back to Step 2.

Step 4. If $i=n+1$, then stop; else, go back to Step 2 .

$L_{1}$ and $L_{2}$ denote the current loads of machine 1 and machine 2 in algorithm Sahni, respectively. And algorithm Sahni first schedules the jobs of $\mathscr{J}^{(1)}$, then schedule $\mathscr{J}^{(2)}$ and so on. The following theorem is from [2].

Theorem 1. If problem P2|pmtn; $d_{j} \mid$-is feasible, then algorithm Sahni gives a feasible schedule.

Theorem 2. Problem P2|pmtn; $d_{j} \mid$-is feasible if and only if it satisfies the following conditions:

(1) $d_{(j-1)}+d_{(i)} \geq \sum_{t=1}^{r_{j-1}} p_{t}+p_{r_{i-1}+1}, i=1,2, \ldots, k, j=$ $1,2, \ldots, i$

(2) $2 d_{(i)} \geq \sum_{j=1}^{r_{i}} p_{j}, i=1,2, \ldots, k$. 
Proof. "only if" part. Problem $P 2 \mid$ pmtn; $d_{j} \mid$-is feasible and denoted by $\sigma$ a feasible schedule for P2|pmtn; $d_{j} \mid$. For any $i$ and any $j$ with $j \leq i$, only $J_{r_{i-1}+1}$ out of $\left\{J_{1}, J_{2}, \ldots, J_{r_{j-1}}\right\} \cup$ $\left\{J_{r_{i-1}+1}\right\}$ can be processed in $\left[d_{(j-1)}, d_{(i)}\right]$ in $\sigma$. Note that $J_{r_{i-1}+1}$ must be completed by $d_{(i)}$ and all the jobs of $\left\{J_{1}, J_{2}, \ldots, J_{r_{j-1}}\right\}$ must be completed by $d_{(j-1)}$. Then we have $2 d_{(j-1)}+\left(d_{(i)}-\right.$ $\left.d_{(j-1)}\right) \geq \sum_{h=1}^{r_{j-1}} p_{h}+p_{r_{i-1}+1}$, which means that (1) holds. Note that all the jobs of all the jobs of $\left\{J_{1}, J_{2}, \ldots, J_{r_{i}}\right\}$ must be completed by $d_{(i)}$. Then we can get that $2 d_{(i)} \geq \sum_{j=1}^{r_{i}} p_{j}$, which means that (2) holds.

"If" Part. When $k=1$, which means that all the jobs have the same deadline $d$. By the conditions, we have $p_{\max }=$ $p_{1} \leq d$ and $\sum_{j=1}^{n} p_{j} / 2 \leq d$. According to the result of [21], we know that there exists a feasible schedule of the $n$ jobs meeting with the same deadline. Now assume that the conclusion holds for $k=t$. We consider the case of $k=$ $t+1$. Let $\overline{\mathscr{J}}=\left\{J_{1}, J_{2}, \ldots, J_{r_{t}}\right\}=\cup_{i=1}^{t} \mathscr{J}^{(i)}$. By the assumption of $k=t$, we know that there exists a feasible schedule $\sigma$ for $\overline{\mathscr{J}}$. Furthermore, by Theorem 1, we can assume that $\sigma$ is generated by algorithm Sahni. Denote by $x$ and $y$ the loads of machine 1 and machine 2, respectively. By the assumption of $k=t+1$ and by the algorithm Sahni, we have that $x+p_{r_{t}+1} \leq$ $d_{(t+1)}$ and $x+y+\sum_{j=r_{t}+1}^{r_{t+1}} p_{j} \leq 2 d_{(t+1)}$. Similar to McNaughton's algorithm of [21], we can schedule the jobs of $\mathscr{J}^{(t+1)}$ starting from $\sigma$ to meet with the deadlines of the jobs of $\mathscr{J}^{(t+1)}$. Then we can get a feasible schedule for the job set $\cup_{i=1}^{t+1} \mathscr{J}^{(i)}$.

Remark 3. Theorem 2 still holds for $d_{(1)} \leq d_{(2)} \leq \cdots \leq d_{(k)}$.

By Theorem 2, we can easily get the following lemma.

Lemma 4. Problem $P 2|p m t n| L_{\max }$ can be solved in $O\left(n^{2}\right)$ time.

In the following, let us consider problem $P 2|\operatorname{pmtn}|\left(L_{\max }^{a}\right.$ : $\left.L_{\text {max }}^{b}\right)$. Let $\left(x_{1}, y_{1}\right)$ and $\left(x_{2}, y_{2}\right)$ be two Pareto optimal points of $P 2|\operatorname{pmtn}|\left(L_{\max }^{a}: L_{\max }^{b}\right)$; then $x_{1}>x_{2}$ means that $y_{1}<y_{2}$. So we can assume $y=f(x)$ such that $(x, y)$ is a Pareto optimal point of $P 2|\operatorname{pmtn}|\left(L_{\max }^{a}: L_{\max }^{b}\right)$, and we know that $y=f(x)$ is a strictly decreasing function on $x$. In order to calculate the tradeoff curve of $P 2|\operatorname{pmtn}|\left(L_{\max }^{a}: L_{\max }^{b}\right)$, we must determine the domain $D$ of $x$ and present an efficient calculation of $f(x)$. Let $t(x)$ be the optimal value of $P 2|\operatorname{pmtn}| L_{\max }^{a} \leq x: L_{\max }^{b}$ then $\left\{(x, t(x)): 0 \leq x \leq x^{0}\right\}$ includes all the Pareto optimal points of $P 2|\mathrm{pmtn}|\left(L_{\max }^{a}: L_{\max }^{b}\right)$; that is, $\{(x, f(x)): x \in D\}$ is the remaining set after $\left\{(x, t(x)): 0 \leq x \leq x^{0}\right\}$ deletes all the non-Pareto optimal points. We call $(x, t(x))$ a transit point if the due date order of the jobs in $\mathscr{J}$ with the due date of $J_{j}^{a}$ considered as $d_{j}^{a}+x-\epsilon$ and the due date of $J_{i}^{b}$ considered as $d_{i}^{b}+t(x-\epsilon)$ are different from the due date order of the jobs in $\mathscr{J}$ with the due date of $J_{j}^{a}$ considered as $d_{j}^{a}+x+\epsilon$ and the due date of $J_{i}^{b}$ considered as $d_{i}^{b}+t(x+\epsilon)$ for sufficiently small $\epsilon$. By Lemma 4 , we first get the optimal value $x_{0}$ of $P 2|\operatorname{pmtn}| L_{\max }^{a}$ : $L_{\max }^{b}<+\infty$ and the optimal value $T_{0}$ of $P 2|\operatorname{pmtn}| L_{\max }^{a}<$ $+\infty: L_{\max }^{b}$ in polynomial time. We simply assume that $x_{0}=$ $T_{0}=0$. If not, we can make $x_{0}=T_{0}=0$ by changing the due dates of the jobs of $\mathscr{F}_{a}$ and $\mathscr{F}_{b}$ to the appropriate numbers.

Obviously, $x \geq 0$. Let $x^{0}$ be the optimal value of $P 2|\operatorname{pmtn}| L_{\max }^{a}: L_{\max }^{b} \leq 0$; then $\left[x_{0}, x^{0}\right]$ is the domain of $x$. We present a strongly polynomial time algorithm to achieve the optimal value of $P 2|\operatorname{pmtn}| L_{\max }^{a}: L_{\max }^{b} \leq 0$. Without loss of generality, for $x \in\{a, b\}$, we assume that the jobs of agent $x$ are ordered by $d_{1}^{x} \leq d_{2}^{x} \leq \cdots \leq d_{n_{x}}^{x}$. Assume that there are total $k_{x}$ different due dates and $d_{(1)}^{x}, d_{(2)}^{x}, \ldots, d_{\left(k_{x}\right)}^{x}$ with $d_{(1)}^{x}<d_{(2)}^{x}<\cdots<d_{\left(k_{x}\right)}^{x}$ are the $k_{x}$ different due dates. Denote by $\mathscr{J}_{x}^{(i)}$ the set of the jobs of due date $d_{(i)}^{x}, i=$ $1,2, \ldots, k_{x}$. Let $r_{0}=0, r_{1}, \ldots, r_{k_{x}}=n_{x}$ be the $k_{x}+1$ numbers such that $\mathscr{J}_{x}^{(i)}=\left\{J_{r_{i-1}+1}^{x}, J_{r_{i-1}+2}^{x}, \ldots, J_{r_{i}}^{x}\right\}, i=1,2, \ldots, k_{x}$. We assume that $d_{0}=0$ and $J_{r_{i-1}+1}^{x}$ are the largest jobs of $\mathscr{J}_{x}^{(i)}$ for convenience, $i=1,2, \ldots, k_{x}$. For any $t \geq 0$, we define $h(t)$ as the smallest number of $\left\{d_{(1)}^{b}, d_{(2)}^{b}, \ldots, d_{\left(k_{b}\right)}^{b}\right\} \cup$ $\{+\infty\}$ strictly larger than $t$. We determine a series of $\delta_{1}$, $\delta_{2}, \ldots, \delta_{v_{0}}$ iteratively, where $v_{0}$ will be determined later. First, we determine $\delta_{1}=\min _{1 \leq i \leq n_{a}}\left\{h\left(d_{(1)}^{a}\right)-d_{(1)}^{a}\right\}$. Then we determine $\delta_{2}=\min _{1 \leq i \leq n_{a}}\left\{h\left(d_{(1)}^{a}+\delta_{1}\right)-d_{(1)}^{a}-\delta_{1}\right\}$. For general $j$, we determine $\delta_{j}=\min _{1 \leq i \leq n_{a}}\left\{h\left(d_{(1)}^{a}+\sum_{k=1}^{j-1} \delta_{k}\right)-d_{(1)}^{a}-\sum_{k=1}^{j-1} \delta_{k}\right\}$. Go on the process until the $v_{0}-$ th iteration such that $d_{(1)}^{a}+$ $\sum_{k=1}^{v_{0}-1} \delta_{k} \geq d_{k_{b}}^{b}$. Then we have $v_{0} \leq k_{a} k_{b} \leq n_{a} n_{b}$. Assume that $\delta_{0}=0$ and $\delta_{v_{0}+1}=+\infty$. We use a bisection search to determine $t_{0}$ such that $P 2|\mathrm{pmtn}| L_{\max }^{a} \leq \sum_{i=1}^{t_{0}-1} \delta_{i}: L_{\max }^{b} \leq 0$ is infeasible and $P 2|\mathrm{pmtn}| L_{\max }^{a} \leq \sum_{i=1}^{t_{0}} \delta_{i}: L_{\max }^{b} \leq 0$ is feasible. And it can be completed in $O\left(\log v_{0}\right)$ implementations of algorithm Sahni. Therefore, we have $\sum_{i=1}^{t_{0}-1} \delta_{i}<x_{0} \leq \sum_{i=1}^{t_{0}} \delta_{i}$. By the definition of $\delta_{t_{0}}$, let $d_{\bar{i}}^{a}=d_{i}^{a}+x$ and $d_{(\bar{j})}^{a}=d_{(j)}^{a}+$ $x, i=1,2, \ldots, n_{a}$ and $j=1,2, \ldots, k_{a}$. We know that $\left\{d_{(\overline{1})}^{a}, d_{(\overline{2})}^{a}, \ldots, d_{\left(\overline{k_{a}}\right)}^{a}\right\} \cup\left\{d_{(1)}^{b}, d_{(2)}^{b}, \ldots, d_{\left(n_{b}\right)}^{b}\right\}$ remains the same increasing order for any $\sum_{i=1}^{t_{0}} \delta_{i}<x<\sum_{i=1}^{t_{0}-1} \delta_{i}$. For $\sum_{i=1}^{t_{0}-1} \delta_{i}<$ $x<\sum_{i=1}^{t_{0}} \delta_{i}$, we apply Theorem 2 to the job set $\mathscr{J}_{a} \cup \mathscr{J}_{b}$ with the deadline $d_{i}^{a}+x$ of $J_{i}^{a}$ and the deadline $d_{j}^{b}$ of $J_{j}^{b}$, $i=1,2, \ldots, n_{a}$ and $j=1,2, \ldots, n_{b}$. Let $\mathscr{J}=\mathscr{J}_{a} \cup \mathscr{J}_{b}=$ $\left\{J_{1}, J_{2}, \ldots, J_{n}\right\}$ and $d_{(1)}, d_{(2)}, \ldots, d_{(k)}$ with $d_{(1)}<d_{(2)}<\cdots<$ $d_{(k)}$ are the distinct deadlines of the jobs of $\mathscr{J}$, where $k=$ $k_{a}+k_{b}$ and $n=n_{a}+n_{b}$. Then $d_{(j)}$ is some $d_{(r)}^{a}+x$ or some $d_{(t)}^{b}$ for any $j$ with $1 \leq j \leq k$. And $p_{i}$ is fixed as some $p_{r}^{a}$ or some $p_{t}^{b}$ for any $x$ with $\sum_{i=1}^{t_{0}-1} \delta_{i}<x<\sum_{i=1}^{t_{0}} \delta_{i}$. So by Theorem 2 , we can get the exact value of $x^{0}$ by solving at most $(k(k+1) / 2)+k$ linear equalities. We demonstrate the idea as two steps.

Step 1. We get the optimal value $T^{0}$ of $P 2|\operatorname{pmtn}| L_{\max }^{a} \leq 0$ : $L_{\max }^{b}$, reset the due date $d_{j}^{b}:=d_{j}^{b}+T^{0}, j=1,2, \ldots, n_{b}$, and keep the due dates $d_{1}^{a}, d_{2}^{a}, \ldots, d_{n_{a}}^{a}$ unchanged. Let $\pi$ be the order of the jobs in $\mathscr{J}$ according to the due dates from small to large. Set $\delta_{1}=\min _{1 \leq i \leq n_{a}}\left\{h\left(d_{1}^{a}\right)-d_{1}^{a}\right\}$. Let $x$ and let $y=u(x)$ be the numbers such that $\mathscr{J}$ has the due date order $\pi$ when the due date of $J_{j}^{a}$ is regarded as $d_{j}^{a}+x$ and the due date of $J_{i}^{b}$ is regarded as $d_{j}^{b}-y$. Consider $d_{j}^{a}+x$ as the deadline of $J_{j}^{a}$ and 
$d_{j}^{b}-y$ as the deadline of $J_{i}^{b}$, applying the Theorem 2, subject to $x+y \leq \delta_{1}$, we can find the exact representation of $u(x)$, where $u(x)$ is a curve of $O\left(k^{2}\right)$ broken lines and the slopes of the broken lines are increasing and $x_{1}$ such that $\left(x_{1}, t\left(x_{1}\right)\right)$ is the transit point in polynomial time.

Step 2. We reset $d_{j}^{b}:=d_{j}^{b}+t\left(x_{1}\right)$ and $d_{i}^{a}:=d_{i}^{a}+x_{1}$, repeat the process of Step 1 to find the next transit point $\left(x_{2}, t\left(x_{2}\right)\right)$, go on the process until we find all the transit points and note that the number of the transit points is bounded by $n_{a} n_{b}$.

In the following, we provide an instance to help the understanding of this algorithm.

Instance. There are two different deadlines $d_{(1)}=3$ and $d_{(2)}=$ 6 and six jobs partitioned into two subsets: $\mathscr{J}^{(1)}=\left\{J_{1}, J_{2}, J_{3}\right\}$ and $\mathscr{J}^{(2)}=\left\{J_{4}, J_{5}, J_{6}\right\}, p_{1}=1, p_{2}=1, p_{3}=3, p_{4}=2, p_{5}=2$, and $p_{6}=6$.

In the instance, we can calculate all the transit points by the algorithm. There are four transit points: $\left(x_{0}, y_{0}\right)=(0,2)$, $\left(x_{1}, y_{1}\right)=(1 / 2,3 / 2),\left(x_{2}, y_{2}\right)=(3 / 2,3 / 2)$, and $\left(x_{3}, y_{3}\right)=$ $(3 / 2,0)$. So the tradeoff curve is demonstrated as Figure 1.

\section{3. $P|\operatorname{pmtn}| L_{\text {max }}^{a}: L_{\max }^{b} \leq Q$}

We now consider problem $P\left|r_{j}, \operatorname{pmtn}\right| L_{\max }^{a}: L_{\max }^{b} \leq \mathrm{Q}$. Without loss of generality, we can assume $Q=0$. Otherwise, we can shift the due date of $J_{i}^{b}$ to some appropriate number, $i=1,2, \ldots, n_{b}$. Similarly, we first deal with the relaxed version of this problem which is to test a trial value of $L_{\max }^{a}$ for feasibility. That is, for a given value $y$, one has to determine whether or not there exists a schedule for which $L_{\max }^{a} \leq$ $y$. This condition is equivalent to the requirement that no job $J_{i}^{a}$ is completed after an induced deadline $d_{i}^{a}+y$ and no job $J_{j}^{b}$ is completed after $d_{j}^{b}$. For the feasibility problem $P\left|r_{j}, \operatorname{pmtn}\right| L_{\max }^{a} \leq y: L_{\max }^{b} \leq 0$, Horn [1] proposed a network flow algorithm to solve it.

Horn' approach is as follows. Suppose $y$ is a trial value for $L_{\text {max }}^{a}$. Let $\left\{e_{1}, e_{2}, \ldots, e_{2 n}\right\}$ with $e_{1} \leq e_{2} \leq \cdots \leq e_{2 n}$ be the ordered collection of release dates $r_{j}^{a}, r_{i}^{b}$ and deadlines $d_{i}^{b}$, induced deadlines $d_{i}^{a}+y$. We arbitrarily break the ties. Further, Define the time interval $E_{k}=\left[e_{k}, e_{k+1}\right]$ for $k=$ $1,2, \ldots, 2 n-1$.

We construct the flow network as follows. There are $n=$ $n_{a}+n_{b}$ job vertices $J_{1}, J_{2}, \ldots, J_{n}\left(J_{j}\right.$ is some $J_{s}^{a}$ or some $\left.J_{r}^{b}\right)$ interval vertices $E_{1}, E_{2}, \ldots, E_{2 n-1}$, a source vertex $S$, and a sink vertex $T$. There ia an $\operatorname{arc}\left(J_{j}, E_{k}\right)$ of capacity $e_{k+1}-e_{k}$ if and only if the release date of $J_{j}$ is no more that $e_{k}$ and $e_{k+1}$ are no more than the (induced) deadlines of $J_{j}$. In addition, there is an $\operatorname{arc}\left(S, J_{j}\right)$ of capacity $p_{j}$ which is the processing time of $J_{j}$ for $j=1,2, \ldots, n$ and an $\operatorname{arc}\left(E_{k}, T\right)$ of capacity $m\left(e_{k+1}-\right.$ $\left.e_{k}\right)$ for $k=1,2, \ldots, 2 n-1$. Now, a maximum value flow is found in $O\left(n^{3}\right)$ time [22, 23]. It should be evident that the trial value $y$ is feasible if and only if the maximum flow value is $P=\sum_{j=1}^{n} p_{j}$, where $p_{j}$ is the processing time of $J_{j}$. If the maximum flow value is indeed $P$, a feasible schedule is easily

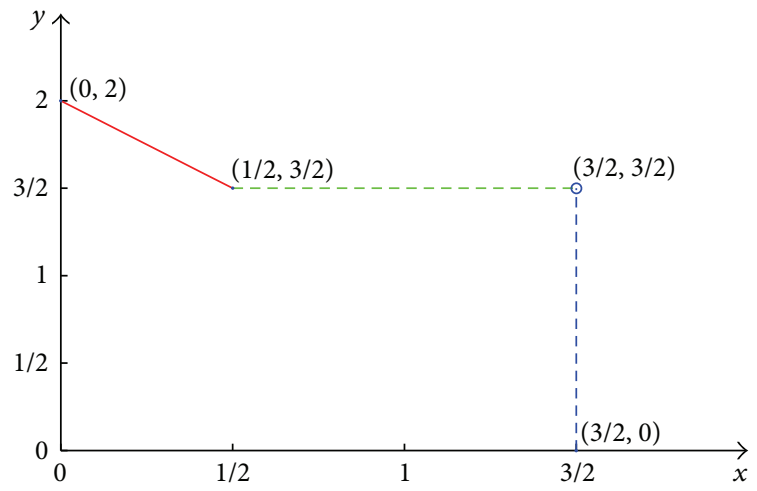

FIgure 1: The solid line and the solid point show all the Pareto optimal points and the dotted line and hollow point are the dominated points.

constructed: for each interval $E_{k}$, the corresponding schedule part can be achieved by applying the McNaughton's algorithm [21]. Notice that there are certain critical trial values of $L_{\max }^{a}$. There are at most $n_{a}\left(n+n_{b}\right)$ values $y$ such that $d_{j}^{a}+y=d_{i}^{b}$ or $d_{j}^{a}+y=r_{k}^{a}$ or $d_{j}^{a}+y=r_{h}^{b}$. The vertex-arc structure of the network remains unchanged for all trial values between two successive critical values.

We propose to find the optimum value of $L_{\max }^{a}$ in two phases. In the first phase, the largest infeasible critical value $y_{0}$ is determined. A bisection search for $y_{0}$ requires the testing of $\log \left(n_{a}\left(n+n_{b}\right)\right)$ trial values or $O\left(n_{a} \cdot n^{2} \log n\right)$ time overall.

In the second phase, a maximum value flow and a minimum capacity cut are found in the network with capacities induced by the value $y_{0}$. Next, a value $y_{1}>y_{0}$ is determined in such a way that the capacity of this cut is increased to exactly $P$. The procedure is then repeated in the network induced by $y_{1}$. This process yields a sequence of increasing trial values $y_{i}$. It terminates when the minimum cut capacity is exactly $P$, that is, at an iteration $z$ where $y_{z}$ is the first feasible trial value and therefore the optimum value of $L_{\max }^{a}$. We will show that $z=O\left(n_{a} \max \{n, m\}\right)$.

Suppose a minimum cut with capacity $P_{0}<P$ is found in the network for $y_{0}$. Consider how the capacity of this cut is changed when $y_{0}$ is increased by some positive amount $\delta$. The capacity $e_{k+1}-e_{k}$ of an $\operatorname{arc}\left(J_{j}, E_{k}\right)$

(a) stays the same if both of $e_{k}$ and $e_{k+1}$ are induced deadlines or not induced deadlines,

(b) increases by $\delta$ if $e_{k}$ is not induced deadline and $e_{k+1}$ is induced deadline, and

(c) decreases by $\delta$ if $e_{k}$ is induced deadline and $e_{k+1}$ is not induced deadline.

A similar situation holds for the capacities of the arcs $\left(E_{k}, T\right)$, except that they change by $m \delta$ or $-m \delta$ rather than by $\delta$ or $-\delta$. All arcs whose capacities are increased are incident with a vertex $E_{k}$ of type (b) of which there are at most $n$. If $\left(E_{k}, T\right)$ is in the cut, then no $\left(J_{j}, E_{k}\right)$ can be forward arc in the cut, so that the cut capacity increases in all arcs incident with $E_{k}$ is at most $m \delta$. It follows that eh capacity of the cut 
is increased by $\mu_{0} \delta$, where $\mu_{0}$ is an integer multiplier with $\mu_{0} \leq n_{a} \max \{n, m\}$. We assert that $\mu_{0} \geq 1$, and let $y^{0}$ be the next critical value after $y_{0}$. Note that The vertex-arc structure of the network remains unchanged for $y_{0}$ and $y^{0}$. Since $y^{0}$ is a feasible critical value, by the max-flow min-cut theorem, we have that $P_{0}+\mu_{0}\left(y^{0}-y_{0}\right) \geq P$, which means that $\mu_{0} \geq 1$. Accordingly, we set $\delta=\left(P-P_{0}\right) / \mu_{0}, y_{1}=y_{0}+\delta$ and repeat.

Each cut in the network can be characterized by a pair $\left(\mu, P^{\prime}\right)$, where $\mu$ is its multiplier and $P^{\prime}$ its capacity. When $y_{l}$ is increased to $y_{l+1}$, the multipliers of cuts do not change, although their capacities indeed do. Suppose that the minimum cut found at iteration $i$ has multiplier $\mu_{i} \geq 1$ and capacity $P_{i}$ and consider the replacement of $y_{i}$ by $y_{i+1}$. Each cut with multiplier $\mu \geq \mu_{i}$ will have its capacity increased to at lease $P$. Hence, $\mu_{i+1}<\mu_{i}$. Note that $\mu_{i} \geq 1$ for all $i$ and $\mu_{0} \leq n_{a} \max \{n, m\}$. It follows that there can be at most $\mu_{0} \leq n_{a} \max \{n, m\}$ iterations.

\section{Conflict of Interests}

The authors declare that there is no conflict of interests regarding the publication of this paper.

\section{References}

[1] W. A. Horn, "Some simple scheduling algorithms," Naval Research Logistics Quarterly, vol. 21, pp. 177-185, 1974.

[2] S. Sahni, "Preemptive scheduling with due dates," Operations Research, vol. 27, no. 5, pp. 925-934, 1979.

[3] S. Sahni and Y. Cho, "Scheduling independent tasks with due times on a uniform processor system," Journal of the Association for Computing Machinery, vol. 27, no. 3, pp. 550-563, 1980.

[4] E. L. Lawler and J. Labetoulle, "On preemptive scheduling of unrelated parallel processors by linear programming," Journal of the Association for Computing Machinery, vol. 25, no. 4, pp. 612-619, 1978.

[5] J. Labetoulle, E. L. Lawler, J. K. Lenstra, and A. H. G. Rinnooy Kan, "Preemptive scheduling of uniform machines subject to release dates," in Progress in Combinatorial Optimization, pp. 245-261, Academic Press, Toronto, Canada, 1984.

[6] E. L. Lawler and C. U. Martel, "Computing maximal "polymatroidal" network flows," Mathematics of Operations Research, vol. 7, no. 3, pp. 334-347, 1982.

[7] C. Martel, "Preemptive scheduling with release times, deadlines and due times," Journal of the Association for Computing Machinery, vol. 29, no. 3, pp. 812-829, 1982.

[8] K. R. Baker and J. C. Smith, "A multiple-criterion model for machine scheduling," Journal of Scheduling, vol. 6, no. 1, pp. 716, 2003.

[9] A. Agnetis, P. B. Mirchandani, D. Pacciarelli, and A. Pacifici, "Scheduling problems with two competing agents," Operations Research, vol. 52, no. 2, pp. 229-242, 2004.

[10] A. Agnetis, D. Pacciarelli, and A. Pacifici, "Multi-agent single machine scheduling," Annals of Operations Research, vol. 150, pp. 3-15, 2007.

[11] T. C. E. Cheng, C. T. Ng, and J. J. Yuan, "Multi-agent scheduling on a single machine with max-form criteria," European Journal of Operational Research, vol. 188, no. 2, pp. 603-609, 2008.

[12] T. C. E. Cheng, C. T. Ng, and J. J. Yuan, "Multi-agent scheduling on a single machine to minimize total weighted number of tardy jobs," Theoretical Computer Science, vol. 362, no. 1-3, pp. 273281, 2006.

[13] J. J. Yuan, W. P. Shang, and Q. Feng, "A note on the scheduling with two families of jobs," Journal of Scheduling, vol. 8, no. 6, pp. 537-542, 2005.

[14] C. T. Ng, T. C. E. Cheng, and J. J. Yuan, "A note on the complexity of the problem of two-agent scheduling on a single machine," Journal of Combinatorial Optimization, vol. 12, no. 4, pp. 387394, 2006.

[15] B. Mor and G. Mosheiov, "Scheduling problems with two competing agents to minimize minmax and minsum earliness measures," European Journal of Operational Research, vol. 206, no. 3, pp. 540-546, 2010.

[16] J. Y.-T. Leung, M. Pinedo, and G. Wan, "Competitive two-agent scheduling and its applications," Operations Research, vol. 58, no. 2, pp. 458-469, 2010.

[17] J. J. Yuan, C. T. Ng, and T. C. E. Cheng, "A note on twoagent scheduling on a single machine with release dates and preemption," Unpublished Manuscript, 2011.

[18] L. Wan, J. J. Yuan, and Z. C. Gen, "A note on the preemptive scheduling to minimize total completion time with release time and deadline constraints," In Submission, 2012.

[19] M. R. Garey and D. S. Johnson, Computers and Intractability: A Guide to the Theory of NP-Completeness, A Series of Books in the Mathematical Sciences, W. H. Freeman, San Francisco, Calif, USA, 1979.

[20] R. L. Graham, E. L. Lawler, J. K. Lenstra, and A. H. G. Rinnooy Kan, "Optimization and approximation in deterministic sequencing and scheduling: a survey," Annals of Discrete Mathematics, vol. 5, pp. 287-326, 1979.

[21] R. McNaughton, "Scheduling with deadlines and loss functions," Management Science, vol. 6, pp. 1-12, 1959.

[22] A. V. Karzanov, "Determining the maximal flow in a network by the method of preflows," Soviet Mathematics Doklady, vol. 15, pp. 434-437, 1974.

[23] R. E. Tarjan, "A simple version of Karzanov's blocking flow algorithm," Operations Research Letters, vol. 2, no. 6, pp. 265268, 1984. 


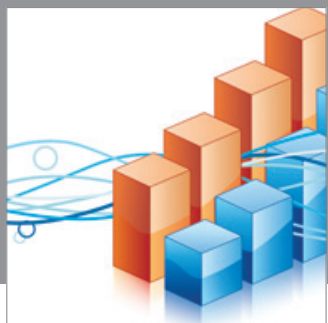

Advances in

Operations Research

mansans

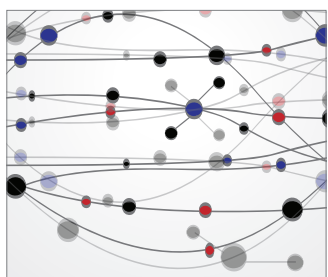

The Scientific World Journal
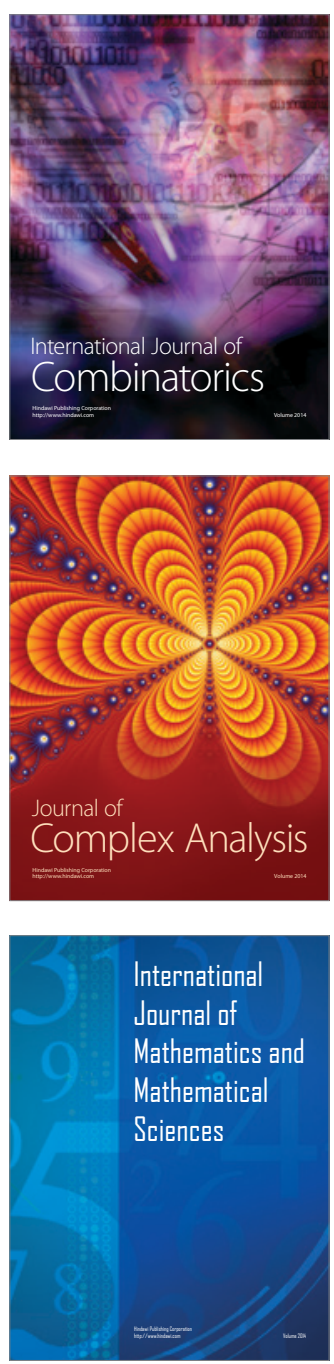
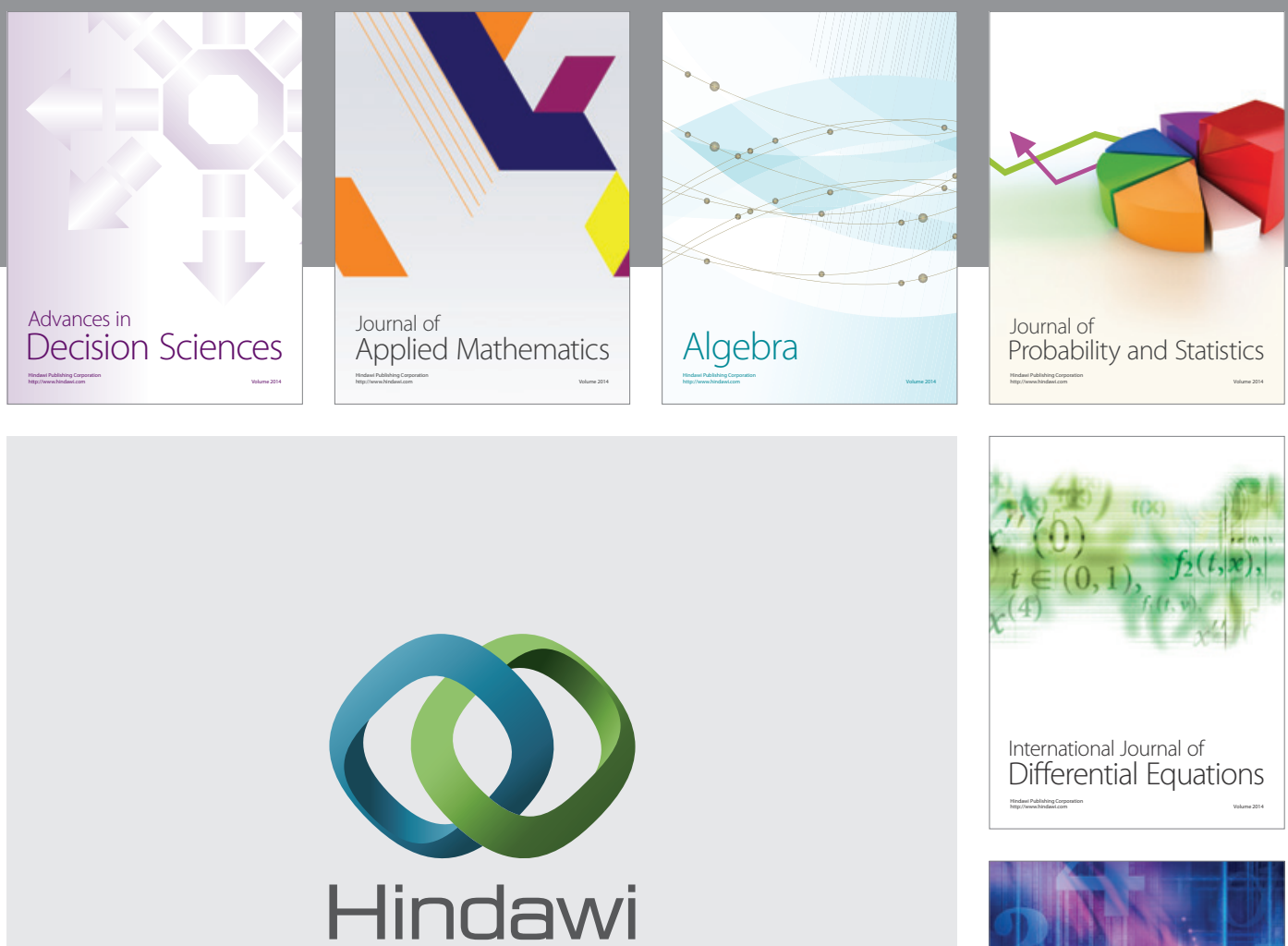

Submit your manuscripts at http://www.hindawi.com
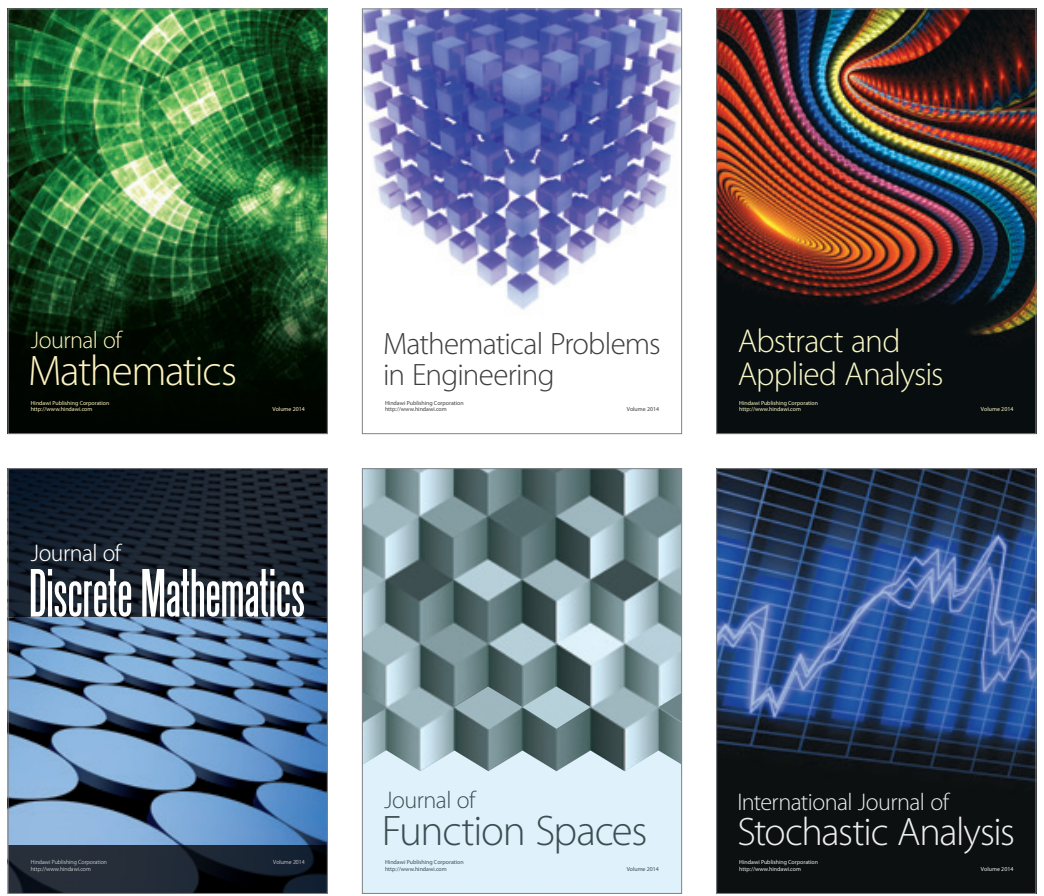

Journal of

Function Spaces

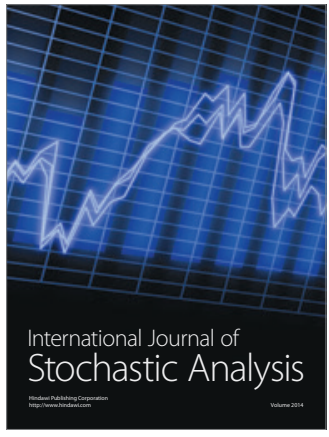

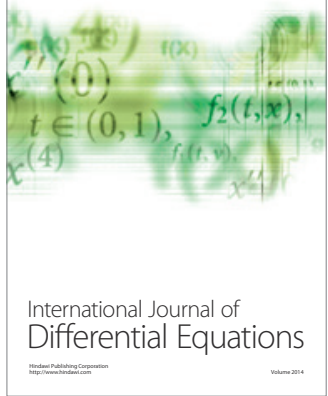
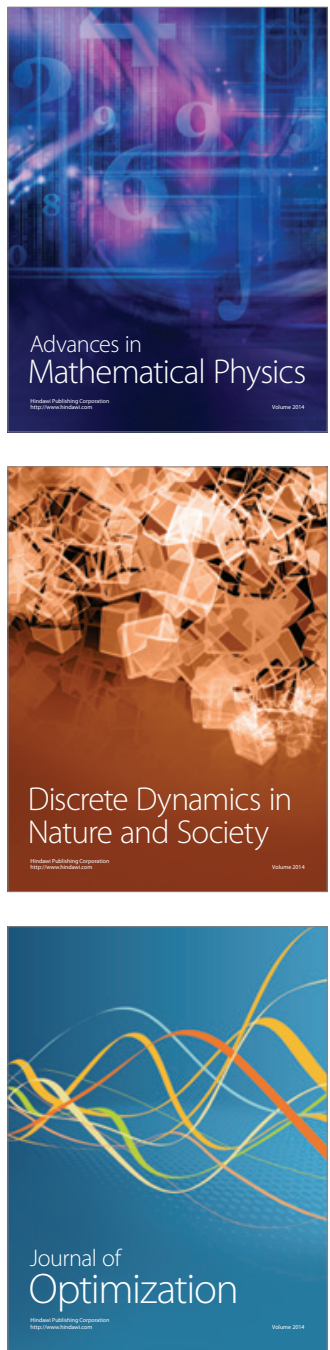